Alzheimer's disease

\section{Lipid lowering agents to delay cognitive decline in Alzheimer's disease?}

\section{F-E de Leeuw}

\section{More work to be done before concluding that lipid lowering agents are useful in Alzheimer's disease}

$\mathrm{T}$ here is conflicting evidence for a causal relation between cholesterol, its treatment, and the incidence of Alzheimer's disease. ${ }^{12}$ Merely the finding of a reduced incidence of Alzheimer's disease among statin users does not necessarily imply any beneficial clinical effect of cholesterol lowering in patients with Alzheimer's disease.

This gap in our understanding of the interplay between cholesterol and Alzheimer's disease prompted Masse and colleagues ${ }^{3}$ to carry out the study on which they report in this months' issue of the journal (see $p 1624$ of this issue).

Previous reports on the effect of statins on the incidence of Alzheimer's disease have always been criticised for possible weaknesses in study designespecially confounding by indication and cessation bias. The authors of the present study have proven to have learned these lessons well by choosing a study design that only included patients with Alzheimer's disease, among whom the majority had already been prescribed lipid lowering agents before the Alzheimer diagnosis was made, a proportion that only increased during follow up. As the investigators mention correctly, this may, at least in part, have avoided indication bias. Other factors that are often associated with indication bias-such as education, severity of the disease, and age-were also equally distributed among the treated and the non-treated groups. The increase in the use of lipid lowering agents along the course of Alzheimer's disease also warrants a good control for another important source of bias-that is, "cessation bias", a phenomenon that may occur if physicians decide not to prescribe relatively expensive drugs any more the moment a patient has become demented. Because of cessation bias the use of lipid lowering agents will be much less among demented patients, consequently leading to spurious associations between lipid lowering agent use and Alzheimer's disease. Perhaps because they rigorously excluded all these biases the investigators succeeded in finding a delay in cognitive decline during the course of Alzheimer's disease among lipid lowering drug users.

Limitations of the study were that the cholesterol lowering effects of the lipid lowering drugs could not be disentangled from other putative effects that these drugs may have had, and that there was no distinction between the various classes of lipid lowering agents (mainly statins and fibrates in their study) with respect to the delay in cognitive decline. Support for at least an important role of statins in this matter comes from a another recent small double blind randomised trial which showed delayed cognitive decline in atorvastatin users compared with a placebo group in patients with Alzheimer's disease. ${ }^{4}$

There are many hypotheses on how lipid lowering agents may work in delaying progression of Alzheimer's disease, and both in vitro and in vivo studies suggest a reduction of cerebral $A_{\beta 1-40}$ and $A_{\beta 1-42}$ by statins. ${ }^{5}$ Another theory, which is not unlikely when dealing with a drug that prevents future vascular events, is a reduction in the cerebrovascular ischaemic events that are known to play a role in Alzheimer's disease. ${ }^{6}$ As Alzheimer's disease has in common with stroke its relatively weak association with cholesterol, a modest beneficial effect of cholesterol lowering on the progression of the two diseases is also a common factor. ${ }^{4}$ Unfortunately the current study does not provide us with detailed longitudinal neuroimaging data that could shed more light on this potential (vascular) underlying mechanism.

As there seem to be so many similarities between Alzheimer's disease and stroke with respect to the "cholesterol" issue, would it not be sensible to include dementia as a primary outcome measure in future cardiovascular trials in order to sort this matter out?

J Neurol Neurosurg Psychiatry 2005;76:1611. doi: 10.1136/jnnp.2005.076315

Correspondence to: Dr F-E de Leeuw, Department of Neurology (HP326), University Medical Centre St Radboud, PO Box 9101, 6500HB Niimegen, Netherlands; h.deleeuw@ neuro.umen.nl

Competing interests: none declared

\section{REFERENCES}

1 Launer $\mathrm{L}$. Demonstrating the case that $A D$ is a vascular disease: epidemiologic evidence. Ageing Res Rev 2002;1:61-77.

2 Zandi PP, Sparks DL, Khachaturian AS, et al. Do statins reduce risk of incident dementia and Alzheimer disease? The Cache County Study. Arch Gen Psychiatry 2005;62:217-24.

3 Masse I, Bordet R, Deplanque D, et al. Lipid lowering agents are associated with a slower cognitive decline in Alzheimer's disease. J Neurol Neurosurg Psychiatr 2005;75:XXX

4 Sparks DL, Sabbagh MN, Connor DJ, et al. Atorvastatin for the treatment of mild to moderate Alzheimer disease: preliminary results. Arch Neurol 2005;62:753-7

5 Fassbender K, Simons M, Bergmann $C$, et al. Simvastatin strongly reduces levels of Alzheimer's disease beta-amyloid peptides Abeta 42 and Abeta 40 in vitro and in vivo. Proc Natl Acad Sci USA. 2001;98: 5856-61, Epub 2001, April 10..

6 De Leeuw FE, Barkhof F, Scheltens P. Alzheimer's disease: one clinical syndrome, two radiological expressions. A study on blood pressure. J Neurol Neurosurgery Psychiatry 2004;75:1270-4.

7 Heart Protection Study Collaborative Group. MRC/BHF Heart Protection Study of cholesterol lowering with simvastatin in 20536 high-risk individuals: a randomised placebo controlled trial. Lancet 2002;360:7-22. 


\section{Cannabinoids in multiple sclerosis: urgent need for long term trials}

\section{J Killestein, B M J Uitdehaag}

The CAMS study represents a substantial step forward, but not the final one

C annabinoids have been suggested for the treatment of numerous conditions, including cancer, vascular, neurodegenerative, and inflammatory diseases. ${ }^{1}$ Probably because of the nature of these substances, there is considerable interest in these drugs from the general public, lay media, and politicians. Frequently, the translation of scientific results is overly optimistic and may have contributed to extensive off label usage of cannabis among chronically ill patients. The evidence for therapeutic efficacy in humans is not as yet convincing.

The CAMS study ${ }^{2}$ by Zajicek and colleagues was the first large symptomatic trial of cannabinoids in multiple sclerosis (MS). In this study, 630 patients with stable MS and muscle spasticity were treated with delta-9tetrahydrocannabinol (THC), cannabis extract or placebo. The main part of the study (covering 15 weeks) is by far the best designed study ever undertaken in this field. There was no evidence of a treatment effect on the primary outcome, the Ashworth scale. The investigators' conclusion, however, was that improvement in mobility and patients' opinion of an improvement in pain might be clinically useful.

In light of these results, the follow up paper by Zajicek (see $p 1664$ of this issue), presenting the data of a 12month, blinded, continuation phase, is most welcome. It is the first study to present longer term data on cannabinoids in MS. Although a small but significant change in Ashworth score was found in the THC group, the clinical significance of this change remains uncertain.

The authors discuss several sources of bias; losses to follow up (around 20\%), discontinuation of medication after the first phase (around 36\%), and the potential for unmasking. The success of masking at the end of follow up is not reported. Even though they discuss that further unmasking was unlikely to occur, the marked degree of unmasking during the main study (up to $70 \%$ ) warrants cautious interpretation of the results, in particular patient derived outcome measures.

The trial was not designed to detect a change in disease activity, but a reduction in relapse associated hospital admissions was suggested during the initial phase. This interesting finding indicates that the anti-inflammatory properties of cannabinoids might play a favourable role in MS disease activity. ${ }^{3}$ However, if confined to relapses classified as serious adverse events, the opposite trend was found during long term follow up.

There were no major safety concerns during the CAMS follow up. Nevertheless, several studies have shown that there are deficits in the performance of complex cognitive tasks in long term cannabis users. ${ }^{4}$ Controversial is the question of whether long term cannabis use can cause irreversible deficits in higher brain function that persist after drug use ceases, ${ }^{4}$ especially in those suffering from diseases such as MS, who are already vulnerable for cognitive decline. In addition, there has been a long standing concern that cannabis use might precipitate mental illness. ${ }^{4}$ In this respect, final results from the CAMS substudy on psychological and cognitive functions are eagerly awaited.

The longer term data indicate that orally administered cannabinoids may hold therapeutic promise as an approach to the treatment of MS related symptoms. However, a balanced assessment of the risk-benefit ratio for cannabinoids in MS is still difficult to make. There is an urgent need for more long term cannabinoid trials in MS using carefully chosen outcome measures. These trials should also focus on different cannabinoid products, including the newer receptor agonists, and different routes of administration. The collection of scientifically sound data will eventually lead to a justified clinical use of cannabinoids, limit the extensive off label usage, and guide the excited scientific and political debate. In that respect, the CAMS study represents a substantial step forward, but not the final one.

J Neurol Neurosurg Psychiatry 2005;76:1612. doi: 10.1136/jnnp.2005.075333

\section{Authors' affiliations}

J Killestein, B M J Uitdehaag, Department of Neurology, VU Medical Centre, Amsterdam,

the Netherlands

Correspondence to: Dr J Killestein, Department of Neurology, VU Medical Centre, De Boelelaan 1117, $1081 \mathrm{HV}$ Amsterdam, the Netherlands; j.killestein@vumc.nl

Competing interests: Both authors participated in a clinical trial of cannabinoids in $\mathrm{MS}$ (Killestein et al. Neurology 2002; 58: 14047), which was financially supported by the Dutch Ministry of Health, Welfare and Sport.

\section{REFERENCES}

1 Klein TW. Cannabinoid-based drugs as antiinflammatory therapeutics. Nat Rev Immunol 2005;5:400-11.

2 Zajicek J, Fox P, Sanders H, et al. Cannabinoids for treatment of spasticity and other symptoms related to multiple sclerosis (CAMS study): multicentre randomised placebo-controlled trial. Lancet 2003;362:1517-26.

3 Pryce G, Baker D. Emerging properties of cannabinoid medicines in management of multiple sclerosis. Trends Neurosci 2005;28:272-6.

4 Iversen L. Long-term effects of exposure to cannabis. Curr Opin Pharmacol 2005;5:69-72. 
Antiepileptic drugs

\section{Withdrawal of antiepileptic drugs in people with non-epileptic seizures}

\section{H A Ring}

The withdrawal of antiepileptic drugs from people with nonepileptic seizures can result in significant benefits

$\mathrm{N}$ on-epileptic seizures (NES) substantially disturb the health and well being of those who suffer from them. In financial terms, the treatment costs of undiagnosed NES may approximate the lifetime costs associated with the treatment of intractable epilepsy. Although difficulties in clarifying the diagnosis are often considerable and the psychological complexity of the condition can appear daunting, ${ }^{1}$ nevertheless significant reductions in medical costs and healthcare utilisation rates have been reported to follow appropriate diagnosis and management of these patients. ${ }^{2}$ Studies that contribute to the improved management of this relatively underresearched condition ${ }^{3}$ are therefore valuable and in the paper by Oto et al (see $p 1682$ of this issue) the important issue of withdrawal of antiepileptic drugs (AEDs) from people with NES is considered.

Initially, a secure diagnosis must be made and the figures reported by Oto et al demonstrate the attendant difficulties. Briefly, of a total cohort of 235 consecutive patients seen in an NES clinic, 184 had a video-EEG confirmed diagnosis of NES and satisfied the authors' criteria for "no epilepsy". Of the 99 patients within this latter group actually taking AEDs, 78 were included in the withdrawal study and in three of these individuals a subsequent diagnosis of epilepsy was made. While revealing that diagnosis is not easy, these figures emphasize that with a careful process the correct diagnosis can be made in a sizeable proportion of patients with suspected NES.

Once the diagnosis has been made, it is important to pay attention to how it is relayed to the patient. If done poorly this can alienate the patient, complicating subsequent management. ${ }^{4}$ Oto et al suggest that the diagnosis is "communicated in a non-judgmental and supportive manner", and this technique is probably an important contributor to the overall success of their approach.

With respect to the benefits of AED withdrawal, as well as removing the burden of inappropriate pharmacological treatment in the great majority of patients and clarifying a diagnosis of epilepsy in a small minority, removing AEDs in people with NES is an important therapeutic manoeuvre in its own right. As the authors note, reductions in NES frequency have been reported in association with various therapeutic approaches and the precise relationship between reduced AEDs and decreased NES remains to be established.
However, clinical experience suggests that for patients with NES, getting an ambivalent message from their doctor "you do not have epilepsy, but we will continue your AEDs" - is likely to undermine psychological treatments and could contribute to increases in anxiety in a patient group for whom anxiety may perpetuate the symptoms of NES.

Oto et al describe a standardized withdrawal protocol that is valuable for several reasons. For clinicians reading the paper it describes a tested approach. For patients (and their GPs) a clearly defined plan will help demonstrate that they are not simply being abandoned following a decision that they do not have epilepsy, but rather that they remain in a controlled therapeutic process, albeit one that has changed direction as new information has come to light. Finally, the authors' recommendation that after withdrawal follow up should be long and careful enough to identify either emergence of epilepsy or other psychological conditions should be emphasized.

J Neurol Neurosurg Psychiatry 2005;76:1613. doi: 10.1136/jnnp.2005.078295

Correspondence to: H A Ring, Developmental Psychiatry Section, University of Cambridge Douglas House, $18 \mathrm{~b}$ Trumpington Road, Cambridge, CB2 2AH, UK; har28@cam.ac.uk

Competing interests: none declared

\section{REFERENCES}

1 Gene-Cos N, Ring HA. Making sense of nonepileptic disorders. Epileptic Disord 2005;7:123-130.

2 Walczak TS, Papacostas S, Williams DT, et al. Outcome after diagnosis of psychogenic nonepileptic seizures. Epilepsia 1995;36:1131-7.

3 LaFrance WC Jr, Devinsky O. The treatment of nonepileptic seizures: historical perspectives and future directions. Epilepsia 2004;45(Suppl 2): 15-21.

4 Shen W, Bowman ES, Markand ON. Presenting the diagnosis of pseudoseizure. Neurology 1990;40:756-9. 